\title{
MODOS DE INTERACCIÓN EN EL DISCURSO INSTITUCIONAL EDUCATIVO SOBRE LAS TIC
}

\author{
MODES OF INTERACTION IN THE \\ INSTITUTIONAL EDUCATIONAL DISCOURSE \\ ABOUT THE ICTs
}

\author{
Lina Marcela LIÑÁN DURÁN \\ Centro de investigaciones semióticas (CeReS). \\ Universidad de Limoges \\ lina-marcela.linan-duran@unilim.fr
}

\begin{abstract}
Resumen: La integración de las tecnologías de la información y la comunicación (TIC) en el campo educativo es una problemática actual sobre la que indagamos desde una perspectiva semiótica. Nos cuestionamos sobre el uso pedagógico de las TIC, interrogando el sentido que construye cada una de las instancias participantes. Nos centramos en el discurso institucional que enmarca la práctica con el fin de identificar la manera en que los establecimientos conciben los procesos de enseñanza-aprendizaje en la era digital y cómo configuran los modos de interacción entre los actores educativos. Buscamos así comprender las dinámicas educativas actuales y posicionarnos frente a ellas.
\end{abstract}

Palabras clave: Sentido. Modos de interacción. TIC. Moodle. Enseñanza universitaria.

Abstract: The integration of the information and communication technologies (ICT) into the educational field is a matter of current questioning that we address from the semiotical perspective. We inquiry about the pedagogic use of the ICT, we examine the sense that each of the participating instances build. We focused on the institutional discourse that frames the practice in order to identify the way in which the establishments 
conceive the processes of teaching-learning within the digital era and how they configure the modes of interaction between the educative actors. We aim to understand the current educative dynamics and to take a position to it.

Key Words: Meaning. Modes of interaction. ICTs. Moodle University teaching.

Frente a la controversia generada por el surgimiento de las TIC y al impacto que estas nuevas tecnologías tienen en la estructura y las maneras de hacer en la escena pedagógica, ha surgido un interés por el uso que de estas se hace en los establecimientos universitarios. En este artículo se presentan algunos resultados del proyecto de investigación doctoral, que se adelanta en el seno del Centro de investigaciones semióticas de la Universidad de Limoges, en el que se busca, desde un enfoque principalmente semiótico, reconocer el funcionamiento de la práctica del uso pedagógico de Moodle en la enseñanza universitaria en Colombia, indagando por el sentido que construye cada uno de los actores que participan en la escena educativa, su interrelación y su confrontación.

Estudiar una práctica social como esta, desde una perspectiva semiótica, se inscribe en el proceso de diversificación de los intereses de esta disciplina hacia nuevos tipos de expresión, nuevos significantes, diferentes a la textualidad. Para la semiótica, el objeto de análisis no está predefinido, sino que es construido por la práctica analítica y por la teoría que la guía (Fontanille, 2016: 19). A través de un procedimiento de modelización se da cuenta de cómo el análisis concreto puede hacer evolucionar la teoría y los modelos establecidos, así como estos últimos surgen de la práctica interpretativa misma. En este sentido:

se supone que el análisis se ajusta al modus operandi de la producción del objeto significante, que en él encuentra y alli se amolda a sus direcciones y a sus articulaciones de tal modo que pueda reconstruir la estructura y explicitarla en un metalenguaje (Fontanille, 2016: 19).

Bajo estos principios se pretende encaminar el estudio del uso de 
Moodle en universidades colombianas.

\section{LA ESCENA PRÁCTICA DEL USO PEDAGÓGICO DE MOODLE}

Atendiendo a la complejidad del objeto de estudio, se optó por abordarlo desde la teoría de los niveles de pertinencia de las prácticas culturales elaborada por Jacques Fontanille. A través de esta propuesta, este investigador busca dar cuenta del recorrido generativo del plano de la expresión del objeto semiótico. Este modelo comprende seis niveles de pertinencia en los que las significaciones culturales pueden expresarse y en el que "cada nivel corresponde a un plano de inmanencia específico" (Fontanille, 2016: 33):

\begin{tabular}{|c|c|c|}
\hline Tipo de experiencia & Instancias formales & Interfaces \\
\hline \multirow{2}{*}{$\begin{array}{l}\text { Figuratividad } \\
\text { [Perceptiva] }\end{array}$} & \multirow{2}{*}{$\begin{array}{c}\text { Signos } \\
\downarrow\end{array}$} & \\
\hline & & Formantes recurrentes \\
\hline \multirow{2}{*}{$\begin{array}{l}\text { Coherencia y cohesión } \\
\text { interpretativas } \\
\text { [interpretativa] }\end{array}$} & \multirow{2}{*}{ Textos-enunciados } & $\begin{array}{c}\text { Isotopias figurativas de la } \\
\text { expresión }\end{array}$ \\
\hline & & $\begin{array}{l}\text { Dispositivo de enunciación' } \\
\text { inscripción }\end{array}$ \\
\hline \multirow{2}{*}{$\begin{array}{l}\text { Corporeidad } \\
\text { [Sensorial] }\end{array}$} & \multirow{2}{*}{$\begin{array}{c}\text { Objetos } \\
+\end{array}$} & Soporte formal de inscripción \\
\hline & & Morfología práxica \\
\hline \multirow{2}{*}{$\begin{array}{l}\text { Práctica } \\
\text { [Vivencial] }\end{array}$} & \multirow{2}{*}{ Escenas prácticas } & Escena predicativa \\
\hline & & Procesos de acomodación \\
\hline \multirow{2}{*}{$\begin{array}{c}\text { Coyuntura } \\
\text { [Situacional] }\end{array}$} & \multirow{2}{*}{ Estrategias } & $\begin{array}{c}\text { Gestión estratégica de las } \\
\text { prácticas }\end{array}$ \\
\hline & & $\begin{array}{c}\text { Iconización de los } \\
\text { comportamientos estratégicos }\end{array}$ \\
\hline $\begin{array}{c}\text { Ethos y } \\
\text { comportamiento } \\
\text { [Etológica] }\end{array}$ & Formas de vida & Estilos estratégicos \\
\hline
\end{tabular}

Tabla 1. Jerarquía de los planos de inmanencia 
Esta jerarquía es composicional, pues cada nivel es necesario para la formación de los demás. Partiendo del principio que la semiótica es una disciplina de investigación que procede por integración, la relación entre los planos de inmanencia está dada por dos operaciones de integración: descendente, que encuentra en el nivel inferior los constituyentes de la forma del nivel superior, y ascendente, que incorpora la unidad inferior en el nivel superior.

En el análisis concreto, la pertinencia del modelo radica en que proporciona las herramientas para elegir el nivel más apropiado para el análisis, según la naturaleza del objeto de estudio y de los objetivos del proceso analítico, así como facilita la integración de elementos de otros niveles, necesarios para el análisis; de la misma manera, amplía los horizontes del análisis al interesarse más en los procesos de construcción y de emergencia de la significación que en sus resultados, es decir, en la significación en acto.

De este modelo, se eligió, entonces, el nivel de las prácticas, que se caracteriza por configurar una escena en la que intervienen diferentes actantes con roles diversos, principalmente modales y pasionales, que se interrelacionan. Así, en la escena práctica del uso pedagógico de Moodle, se han identificado tres actores principales: los usuarios, es decir, estudiantes y profesores, la plataforma y la institución.

Para entender la significación de esta práctica, es necesario realizar las dos operaciones de integración. Ubicados en el nivel de pertinencia de las prácticas, se realiza una integración descendente que vincula el nivel inmediatamente inferior, el de la plataforma Moodle como el objetosoporte. Y una integración ascendente del nivel de las estrategias que alude a las estrategias puestas en escena tanto por las instancias institucionales para regular la práctica como por los usuarios mismos en la acomodación estratégica que llevan a cabo para gestionar las diferentes prácticas que intervienen en la escena del uso pedagógico de Moodle.

En lo que se refiere a la segunda integración, la que vincula la instancia institucional a la práctica estudiada, se considera pertinente recurrir a otro modelo semiótico que ayude a elucidar la construcción de la significación en las relaciones configuradas por la institución entre los actores participantes. Se trata de la teoría de los regímenes de interacción de Éric Landowski (2005). 


\section{REGÍMENES DE INTERACCIÓN}

Las reflexiones de Éric Landowski (2005), guiadas en dirección del sujeto y su relación con el mundo y con los otros sujetos, lo han llevado a la creación de un modelo que articula cuatro regímenes de interacción, a saber: la programación, la manipulación, el ajuste y el accidente. Cada uno de ellos, está fundado en un principio semiótico, respectivamente: la regularidad, la intencionalidad, la sensibilidad y el riesgo. Los dos primeros fueron retomados de la teoría narrativa clásica y reformulados en términos de intersubjetividad. Grosso modo, la programación adopta la estrategia de la seguridad y consiste en la previsión de estrictas regularidades de comportamiento en el recorrido de los sujetos configurándolos como no-sujetos, mientras que en la manipulación los sujetos llevan a cabo procedimientos persuasivos frente a sus interlocutores con el fin de hacerlos hacer modificando sus competencias modales. En estas circunstancias, a diferencia de la programación, incluso si está la intención de ejercer una influencia en el otro para llevarlo a la acción, en la manipulación se le atribuye un estatus semiótico de sujeto.

Frente a estos dos regímenes, en los que existe una influencia en el otro, este investigador propone otros dos: el accidente y el ajuste. El primero, cuyo principio fundador es el riesgo, invita a los agentes a establecer relaciones imprevisibles. Ahí hay un lugar para lo inesperado, lo que representa una clara toma de riesgo. Respecto al ajuste, este se caracteriza por una igualdad entre sujetos que está basada en la sensibilidad de cada uno. En relación con los otros tres, en este régimen, el comportamiento del sujeto no puede ser previsto, "obedece a una dinámica propia" (Landowski, 2005: 40) porque la finalidad es la realización mutua de los sujetos en la interacción. Estas cuatro categorizaciones se interdefinen y forman un sistema: 


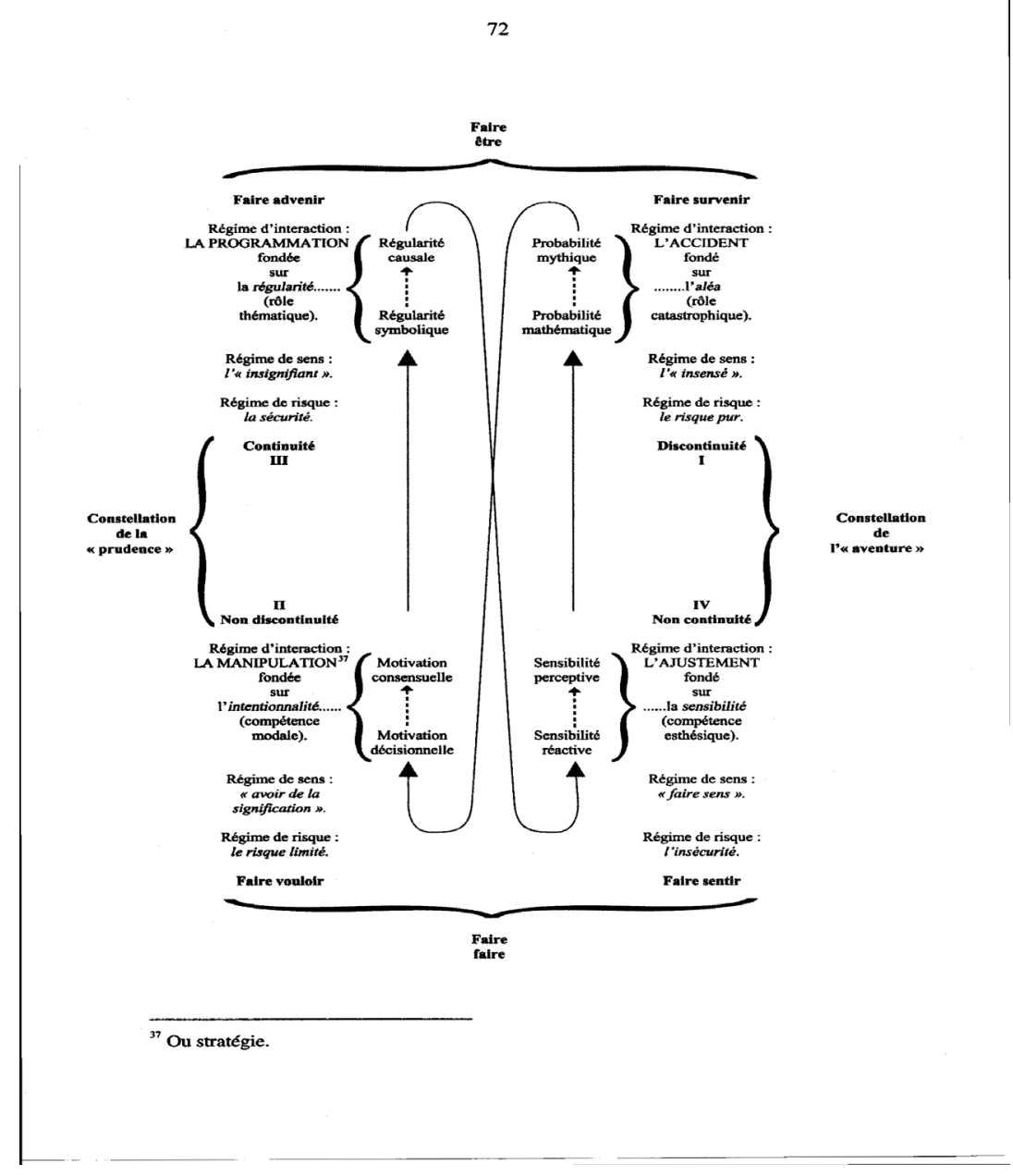

Figura 1. Regímenes de interacción

\section{CONSTITUCIÓN DEL CORPUS}

Acceder de manera directa a las prácticas o a las formas de vidas, que Fontanille ubica en los últimos niveles de su modelo, representa una gran dificultad. Por esta razón, es necesario admitir un cierto grado 
de intermediación recurriendo a datos indirectos, a diferentes capas, a diferentes elementos complementarios que ayuden a compensar la ausencia de los datos directos.

El reconocimiento de los tres actores principales que intervienen en la escena práctica del uso pedagógico de Moodle lleva a cuestionarse cómo la práctica es configurada desde cada una de estas perspectivas. Así, se hace necesario la construcción del corpus que resulta heterogéneo debido a que se constituye de diferentes sistemas de significación de naturaleza textual y multimodal. Se cuenta, entonces, con:

a) las políticas institucionales y algunos documentos del Ministerio de Educación Nacional que enmarcan el uso de TIC en las universidades;

b) las interfaces de la plataforma Moodle, personalizadas por cada institución, con el fin de estudiar las posibilidades de recorrido y de trabajo que ofrece la plataforma a los usuarios a través de su diseño;

c) transcripciones de las entrevistas realizadas a estudiantes, profesores y administrativos de cada institución educativa sobre su concepción de las TIC y su experiencia de uso de estas tecnologías porque en la búsqueda de la comprensión del sentido de una práctica social, es importante poder aprehender la experiencia vivida por los actores que participan en ella.

Sin embargo, a causa de algunos factores institucionales y de otros intrínsecos a la misma práctica, no fue posible un acceso directo a la realización de la acción. No pudiendo acceder a lo vivido de la experiencia, se optó, entonces, por el discurso de la experiencia vivida. Lo que posibilita, por ejemplo, acceder a lo que ha sido realmente importante para el sujeto, pues él es "capaz de recuperar reflexivamente y de restituir para sí mismo y, dado el caso, para otros aquello que antes ha hecho sentido en la vivencia de la experiencia, es decir, en la captación inicial" (Landowski, 2011: 152).

\section{EL USO DE MOODLE DESDE LA PERSPECTIVA INSTITUCIONAL}

Para este artículo, se ha elegido estudiar el discurso institucional. Efectuando la integración ascendente, se reconoce que el uso pedagógico de Moodle no se limita a que los usuarios accedan a la plataforma introduciendo su nombre de usuario y su contraseña, ni a que utilicen las 
herramientas y los recursos que ella propone. Es también la elección de la plataforma y de la modalidad de enseñanza en la que se utiliza, así como el conjunto de condiciones, normas y disposiciones que regulan su uso en el marco de uso de las nuevas tecnologías, y que emanan de las instancias institucionales, lo que se conoce como dispositivos de uso institucionales.

Por institución, se va a designar "una clase hipotética de instancias reguladoras que, en el ejercicio de sus funciones de regulación, toman específicamente por objeto las interacciones, virtuales o efectivas, inscritas por hipótesis en el nivel micro-social e intersubjetivo" (Landowski, 1993: 111). Es, entonces, en el orden de lo macro-social donde se revela la problemática institucional que, siendo más global, enmarca la de las interacciones entre los sujetos.

Los establecimientos de educación superior se erigen como dispositivos de organización y de gestión de los procesos educativos, y en este caso, como sistemas que fijan el marco de uso de las TIC en la experiencia pedagógica. Desde una perspectiva semiótica, se trata en efecto de la figura actancial del destinador que busca hacer actuar a los sujetos, condicionando sus roles, sus interacciones con las herramientas digitales y con los otros actores educativos.

Concebidas como figuras de poder, estas instancias, al momento de configurar el dispositivo de uso, suscriben una intención determinada, que se propone examinar a continuación, a partir de las construcciones discursivas de los sujetos administrativos, encargados de los servicios TIC en las cinco universidades que hacen parte de este estudio.

De todos los elementos que constituyen la instancia institucional, se opta por centrarse en el discurso de los cinco sujetos administradores, quienes, posicionados en su campo semiótico, vehiculan un saber relativo al mismo objeto, las TIC, que es construido gracias a la puesta en relación de este objeto con otros contenidos discursivos como la comunicación, la mediación, la autonomía, los procesos de aprendizaje, la evaluación, la investigación, la políticas de integración, los usuarios, la gestión del tiempo, el diseño, la plataforma Moodle, la resistencia, entre muchos otros, con el objetivo de mostrar sus características y de hacer una primera valoración. Sin embargo, la mayoría de predicaciones no se quedan en el nivel del saber sino que comprometen el creer, pues los enunciadores asocian las tecnologías digitales no solo a objetos como los ya mencionados, sino también a otros sujetos y asumen una posición comprometiendo su sistema 
de creencias, lo que implica una confrontación entre universos de asunción (Fontanille, 2003: 236).

Entre los procedimientos cognitivos llevados a cabo por los enunciadores, identificamos también su estrategia de estructuración ${ }^{1}$. Incluso si la situación de entrevista podía representar una restricción respecto a las temáticas y las preguntas previstas por el entrevistador para la discusión, en general eran los entrevistados quienes orientaban la conversación realizando operaciones de asociación, comparación, categorización respecto al objeto TIC.

Como actantes intencionales, los enunciadores ponen en escena un hacer-saber cumpliendo un cierto número de operaciones cognitivas identificables en sus construcciones discursivas, que serán analizadas con el objetivo de identificar: (a) cómo los establecimientos conciben las TIC en los procesos de enseñanza-aprendizaje y (b) cómo configuran los modos de interacción entre los actores educativos.

\section{ETAPAS DE LA RECONSTRUCCIÓN DE REPRESENTA- CIONES SOBRE LAS TIC}

En el proceso de reconstrucción de estas representaciones, se identifica la recurrencia de tres momentos en la estructura de sus discursos. El primero es en el que bosquejan su posición personal e institucional sobre las TIC; en el segundo, en el que justifican explícitamente por qué integrarlas en el campo educativo; y en el tercero, revelan cuál es el propósito con el que utilizan estas tecnologías en clase, mostrando cuál es realmente el lugar que les otorgan en los procesos de enseñanza y de aprendizaje.

En términos generales, en un primer momento, todos los enunciadores orientan sus discursos hacia definiciones generalistas que pueden resumirse en dos grupos. Por un lado, quienes las conciben explícitamente como una mediación, y, por el otro, quienes las definen como herramientas de apoyo. En el primer caso, la definición se hace en función de la posición que ellas ocupan en la escena pedagógica, así la relación educativa que integra el uso de tecnologías obedece a una sintaxis figurativa que requiere de actantes posicionales, donde las TIC juegan el

${ }^{1}$ Entrada Punto de vista del glosario de semiótica de Bertrand (2006: s. p.). 
rol de actantes de control dado que ellas gestionan la interacción entre la fuente y el blanco, es decir, entre el profesor y el estudiante, y también modalizan el hacer de ambos actores entre otros aspectos, por el grado de presencia de estas que está dado por la modalidad de enseñanza elegida: presencial, híbrida o a distancia. Asimismo, ese rol mediador atribuido a las TIC es comprendido de forma diferente según la posición axiológica de las tres instituciones. En el segundo, estas nuevas tecnologías son definidas no por la posición que ocupan sino por el rol que juegan en la escena pedagógica, pensada como una sintaxis narrativa en la que las TIC intervienen como el ayudante que "aporta su ayuda a la realización del programa narrativo" (Greimas et Courtés, 1993: 10), en este caso, a la consecución de objetivos de aprendizaje por parte de los estudiantes o un acompañamiento eficaz a los estudiantes por parte del profesor.

En el segundo momento, los sujetos de la enunciación justifican el uso de las tecnologías digitales en clase. Un cierto número de las razones expuestas hacen alusión a lugares comunes, a lo que con frecuencia se reduce la reflexión sobre los usos y los desafíos educativos de lo digital, según Philippe Meirieu (2012: 159), especialistas en Ciencias de la educación. Siguiendo las propuestas de Andrea Semprini (1994: 7), quien revisita el concepto de lugar común desde una perspectiva semiótica, se considera que estos son lugares comunes fundados en saberes dóxicos, circunstanciales que gracias a su carácter vago e indefinido permiten su apropiación situada y su movilización por parte de los actores implicados en la interacción para producir un acuerdo compartido tanto en la situación de entrevista como en las relaciones que se tejen con los otros actores educativos, como los profesores y los estudiantes. Por ejemplo, al recurrir a la noción de autonomía, los sintagmas ciudadanos del ciberespacio y nativos digitales, los enunciadores revelan algunas de las características del lugar común, principalmente lo que Semprini llama su fuerza instituyente, porque imponiendo esta competencia a los sujetos y refiriéndose a ellos con estos apelativos, los enunciadores buscan instituir la identidad de los sujetos, en particular de los estudiantes, comprometidos en las prácticas educativas actuales.

En un tercer momento, los enunciadores exponen cuál es su propósito al integrar las TIC en las prácticas educativas, proporcionando nuevas

${ }^{2}$ Entrada del Diccionario "Enunciado". 
pistas relacionadas con las políticas de integración y con las interacciones entre los actores que concretiza la reconstrucción de las representaciones. Algunos sujetos de enunciación que habían definido las TIC como ayuda o mediación, agregan que en los establecimientos a los que ellos pertenecen, las tecnologías digitales son solo un complemento a las clases presenciales, y que así está definido en las políticas institucionales. Otros ven en las TIC una herramienta de seguimiento y control tanto de los procedimientos de integración de tecnologías como del hacer de profesores y estudiantes. Para otros, en el marco de la enseñanza a distancia, además de fortalecer la autonomía en los estudiantes, las TIC garantizan un acompañamiento más constante y una cierta presencia de los profesores gracias, principalmente, al contacto sincrónico que permiten.

\section{CONFIGURACIÓN DE LAS INTERACCIONES ENTRE LOS ACTORES EDUCATIVOS}

Examinando los discursos de los cinco enunciadores, se ha podido reconstruir las representaciones que elaboran sobre las nuevas tecnologías. Dichas representaciones orientan la configuración de las prácticas de uso de las tecnologías, especialmente de la plataforma Moodle, y de las interacciones entre los actores que participan. Para corroborarlo, el enfoque de la sociosemiótica, tal y como fue elaborado por Eric Landowski, se revela pertinente, principalmente porque desde su punto de vista, "en el campo educativo, es justamente la cuestión del sentido de las prácticas y más precisamente del sentido de las prácticas de interacción que constituye el núcleo de los problemas sociales" (Landowski, 2015: 2).

Para este investigador, nuestra percepción eufórica o disfórica del mundo está asociada a "la diversidad de los regímenes de presencia y de interacción en los cuales se inscriben nuestras relaciones con el mundo y con los otros" (Landowski, 2005: 12). Dicho de otro modo, existen principios semióticos más o menos recurrentes que permiten la construcción de una tipología de regímenes de interacción para explicar la naturaleza de las relaciones entre los sujetos. En sus predicaciones, el personal administrativo se manifestó respecto al uso de TIC y, por lo tanto, a las relaciones que ellos, como institución, mantenían con los profesores, así como a la manera en que ellos esperaban que los profesores interactuaran con sus estudiantes. A partir de los resultados de los análisis anteriores, se 
constata la existencia de tres modos de interacción que corresponden a las representaciones identificadas. Modos que buscan orientar los recorridos de acción de los sujetos usuarios de TIC en la escena pedagógica. A saber, la programación, la manipulación y el ajuste. Estos tres modos o regímenes están presentes en todos los discursos en grados diferentes, pero en cada institución hay uno que predomina.

\subsection{La programación}

En la muestra analizada, este modo de interacción se caracteriza básicamente por a) la búsqueda de un régimen de seguridad, b) por basarse en una lógica de substitución y c) por la imposición de roles temáticos a los sujetos en los recorridos que se les proponen.

La configuración hecha por dos de las instituciones está fundada en el régimen de la programación, pues las consideran como un medio de sistematización de la actividad pedagógica o de seguimiento de las acciones realizadas por los actores educativos. Buscan controlar todo con el fin de sentirse en seguridad limitando los riesgos que puede conllevar la interacción con el otro. Estos dos enunciadores administrativos optan por la elección de una plataforma digital —Moodle - que medie la relación profesor-estudiante y les permita conservar una trazabilidad de las acciones realizadas por los usuarios con el fin de vigilar lo que hacen. En este escenario, no hay espacio suficiente para una relación espontánea entre profesor y estudiante porque es necesario que todo esté previsto con antelación, incluso el discurso del profesor porque como todo está grabado, dice uno de los enunciadores, es difícil corregir lo que ya se dijo o se escribió, ya los profesores no son libres de expresarse como ellos quieren, de ser naturales (Alexander, 2018: 62), so pena de someterse a "acciones correctivas" (Alexander, 2018: 71), que consisten en hacerles unas formaciones y un seguimiento individual hasta lograr que su hacer se adapte a lo que ya está estipulado.

Estos establecimientos prescriben recorridos de acción precisos, lo que, siguiendo las propuestas de Nicole Pignier, nace de una intención operacional (2016a: 1), construida sobre la base de un modo de organización tecnocrático porque se privilegian las competencias técnicas antes que la reflexión sobre los desafíos axiológicos y pedagógicos que implica la integración de las tecnologías digitales en el aula de clase. 
Las representaciones que los sujetos de enunciación de estos dos establecimientos tienen sobre la apropiación de las nuevas tecnologías en la educación, construyen, en el seno de estas instituciones, dinámicas de uso individual y colectivo que el discurso social, principalmente, mediático, transforma en recorridos de acción estereotipados. Ellos eligieron incorporar estas tecnologías en las prácticas de enseñanza-aprendizaje imponiendo algunas formas de uso apoyadas en ideas compartidas socialmente, seguros de que los destinatarios de estos itinerarios van a conformarse convencidos de la pertinencia de sus propósitos, como si "estos hicieran parte del orden natural de las cosas" (Landowski, 2005: 31).

Estas dos posiciones se fundan en una lógica de substitución que "expresa una percepción de las tecnologías digitales como la vía mediática por excelencia" (Pignier, 2016b: 8). En ambas universidades, inicialmente concebían las TIC como un complemento a la modalidad presencial, pero esa idea se transformó en tener verdaderos cursos virtuales (Alberto, 2018: 10) porque, según ellos, la enseñanza a través de interfaces digitales se impone cada vez más por razones de tiempo, de organización y porque su uso favorece el desarrollo de la autogestión del conocimiento en los estudiantes (Alexander, 2018: 64). Asimismo, tener cursos completamente virtuales es, según los enunciadores, "posible y necesario" (Alberto, 2018: 5) porque muestra el estado de madurez de los profesores, su interés en el aprendizaje de sus estudiantes y permite a las instituciones tomar riesgos al aumentar el grado de presencia de las TIC en clase.

Asimismo, los vínculos entre la institución, el profesor y el estudiante se construyen sobre la modalidad deóntica, lo que parece reducir a los sujetos-usuarios a simples ejecutantes. Se les reconoce la facultad de hacer, pero esta está semánticamente determinada por la atribución de roles temáticos que circunscriben sus esferas de acción incluso contra su voluntad. Es el caso de una de las instituciones donde a pesar de que los estudiantes se oponían, los administrativos les impusieron cursos virtuales, a los cuales "ellos se fueron acostumbrando" (Alexander, 2018: 61). En esta misma universidad, según el enunciador, se hace una comparación constante entre los profesores que usan TIC y los que no. El trabajo de los primeros es destacado porque consiste en ir más allá de los contenidos valorando cómo el estudiante hace autogestión del conocimiento, mientras que los segundos son configurados como sujetos que se limitan a calificar sin preocuparse por la comprensión de los aprendices y que, por lo tanto, 
deben cambiar completamente su mentalidad, pensar estratégicamente, aprender a manejar las herramientas de comunicación, etc.

\subsection{La manipulación}

En contraste con la lógica de la programación, se identifica un segundo modo de interacción: el de la manipulación. En una de las universidades predomina la puesta en marcha de una estrategia que orienta principalmente las interacciones de la institución y los profesores, y también el vínculo entre estos últimos y los estudiantes. Este segundo régimen de interacción se caracteriza a) por la atribución de una competencia modal a los sujetos-usuarios y b) por poner en escena estrategias para logar el hacer querer en los sujetos.

La institución, reconociéndoles el estatus compartido de sujetos, supone que los usuarios estudiantes y profesores poseen una competencia modal, es decir, una competencia semiótica que los habilita a comunicar $\mathrm{y}$, por este hecho, los hace influenciables los unos a los otros. En esta situación, el objetivo del establecimiento es hacerles "ejecutar un programa dado" (Greimas \& Courtés, 1993: 220)․․ Así, el enunciador, configurado como destinador, en nombre de la institución propone un contrato a los docentes - el destinatario-sujeto - ofreciéndoles varios cursos de formación y les otorga el derecho de decidir si quieren o no participar. Sin embargo, con el fin de ampliar la participación, se pone en marcha lo que él llama el programa de implementación de la política TIC en tres niveles, cuya primera etapa es crear una cultura de uso de TIC. Se trata de presentar la plataforma Moodle a los profesores, todo lo que esta propone: las herramientas, los recursos, las actividades, etc. El sujeto de enunciación explica que en su universidad comprendieron que si quieren que el programa funcione no deben obligar a los profesores sino más bien hacer que ellos quieran usar la plataforma.

La estrategia implementada consiste en la creación de un grupo de trabajo, compuesto por un profesor representante de cada facultad, cuya función es alentar a los otros docentes a integrar las TIC en sus clases. Para lograrlo, se esfuerzan en moldear su competencia al informarles sobre las ventajas que ofrece Moodle para la práctica educativa, las experiencias

3 Entrada del Diccionario "Manipulación”. 
exitosas de sus colegas y los beneficios económicos que ellos pueden obtener de parte de la institución, porque, como afirma Landowski, "para hacer que un sujeto 'quiera' hacer algo, es necesario, en primer lugar, hacerle creer o hacerle saber que tendrá beneficios al quererlo" (Landowski, 2005: 41-42). Proponiéndoles objetos de valor positivo, la institución recurre a la manipulación por tentación. Una vez obtenida la adhesión de los docentes, esta se compromete a proporcionar las condiciones necesarias para lograr los objetivos de integración de TIC.

\subsection{El ajuste}

En el análisis de la muestra se encuentra un tercer régimen en el que, tal como en la manipulación, los actores son considerados como verdaderos actantes sujetos. Se trata del ajuste, en el que se conciben las relaciones entre sujetos como procesos interactivos en los que los comportamientos no son previsibles, por el contrario, cambian según la diversidad de las situaciones vividas. Aquí la influencia de los unos sobre los otros pasa primero por el contacto entre los cuerpos sintientes en interacción. Los lazos establecidos entre los actores educativos en las otras dos instituciones se inscriben en este régimen. Pese a que en uno de los establecimientos se ofrece formación presencial, $y$, en el otro, programas a distancia, ambos se caracterizan por basarse en una lógica de la complementariedad y por fundar las interacciones entre los actores en el hacer sentir (Landowski, 2005: 44).

En los programas presenciales, antes de que los profesores comiencen a utilizar la plataforma, reciben una formación de dos años, que consiste en primer lugar en una reflexión entre colegas sobre la práctica pedagógica enfocada en, por una parte, reorganizar los contenidos de los cursos en función de lo que es realmente esencial para los estudiantes y, por otra, de proponer nuevas metodologías que necesiten o no de las TIC. Esta reflexión es compartida por el enunciador que dirige los programas a distancia, quien afirma que para la "apropiación y transformación del aula [...] se requiere no una formación en uso de herramientas, sino un diálogo, unas reflexiones, un empoderamiento de las tecnologías" (Beatriz, 2018: 38). En contraste con el modo de interacción de la programación donde predomina el discurso tecnocrático, bajo el régimen del ajuste, se privilegia una organización centrada en el cuestionamiento y la reflexión. 
De la misma manera, la relación entre profesores y estudiantes emerge de una intención perceptiva (Pignier, 2016a). Por ejemplo, en los cursos presenciales, el primer día de clases los docentes proponen el plan de desarrollo de curso a los estudiantes, quienes pueden discutir al respecto sin la presencia del profesor, para luego hacerle parte de sus comentarios y sugerencias. Ellos construyen juntos este plan porque representa un trabajo de "compromiso mutuo" (Cecilia, 2018: 23).

Por otra parte, con el uso de tecnología en clase, al comienzo siempre hay dificultades de comprensión, pero "eso se va acomodando con el tiempo, porque el profesor tiene que aprender a pensar como el estudiante. Y el estudiante también como el profesor. Van mutuamente aprendiendo" (Cecilia, 2018: 25). Se mantiene la dinámica de hacer juntos (Landowski, 2005: 42), de hacer con el otro, lo que hace posible la construcción del mundo que los rodea como mundo significante.

En ambas instituciones se sigue una lógica de complementariedad en cuanto al uso de tecnologías en clase. Al considerarlas más como un medio que como un fin en sí mismas (Pignier, 2016b: 8), tanto en la formación a distancia como en la presencial, se recurre a ellas para crear dispositivos que mejoren las experiencias de enseñanza y de aprendizaje. En el primer caso, las TIC se integran a la escena pedagógica con el fin de crear una presencia de los docentes, y de equilibrar el desfase espacio temporal en las interacciones, proponiendo, a través de las plataformas Moodle y WiziQ, clases sincrónicas durante todo el día, como se hace en la presencialidad. En el segundo caso, las tecnologías son vistas como un ayudante en el desarrollo de las clases y, especialmente, en la comunicación y comprensión entre profesores y estudiantes.

Esta configuración de las relaciones entre interactantes, que se funda en el reconocimiento de la sensibilidad del otro, obedece a una lógica de presencia sensible. En la universidad donde se proponen formaciones presenciales, el enunciador aclara que los estudiantes no prefieren los cursos completamente virtuales por razones idiosincráticas. Están de acuerdo con el uso de TIC en clase, pero también necesitan sentir la presencia del profesor porque, como lo afirma el enunciador: "los latinos somos una cultura de mucha piel, de mucho contacto" (Cecilia, 2018: 23) y los estudiantes son el producto de esa cultura.

En lo que respecta los programas a distancia, la universidad reconoce que la presencia es muy importante. Entonces, el servicio de 
Bienestar Universitario crea un proyecto que consiste en la realización de actividades de integración en cada una de las regiones que pertenecen al programa, con el fin de que los estudiantes se conozcan, sepan que no están solos, se sientan miembros de una comunidad y, en adelante, vean las clases como "un momento importante de interacción y de reconocimiento del otro, y de construir con el otro" (Beatriz, 2018: 39). Estos encuentros ofrecen la posibilidad de sentirse reciprocamente y favorecen la creación de redes que se vuelven fundamentales para el éxito del proceso individual, para evitar, por ejemplo, el abandono.

\section{A MODO DE CONCLUSIÓN}

A través de los análisis realizados, recurriendo tanto al modelo de las prácticas culturales como al de los regímenes de interacción, se han identificado las representaciones que las cinco universidades colombianas, que participan en este estudio, tienen sobre las TIC y la correspondiente configuración de las interacciones entre los actores educativos y de sus recorridos de acción que ellas elaboran.

Las TIC son herramientas:

$\square$ De seguimiento, control y modalización del hacer de los usuarios, para dos de los establecimientos, quienes inscriben sus relaciones con los otros sujetos en el modo de la programación.

$\square$ De ayuda y apoyo a las clases, para otras dos universidades, quienes reconociendo a los otros como sujetos e interesándose más por el proceso de los estudiantes que por el uso de la herramienta, configuran sus interacciones sea en el régimen de la manipulación o en el del ajuste.

$\square$ Que facilitan la presencia del profesor y las interacciones con los estudiantes, en los programas a distancia de la quinta universidad, donde el interés por el sentir de los sujetos, los lleva a inscribir sus prácticas en el modo de interacción del ajuste.

Identificar las representaciones sobre las TIC y la configuración de las interacciones entre los actores educativos, permite entender cómo se construye la significación alrededor de estas tecnologías y cómo las instancias institucionales orientan las experiencias de enseñanza y de 
aprendizaje en las que se hace uso de la plataforma Moodle. Estos elementos característicos del discurso del actor institucional son puestos en relación con la configuración realizada por los otros dos actores de la escena del uso pedagógico de Moodle: la plataforma, a través de su diseño, y los usuarios, profesores y estudiantes, en el uso efectivo que hacen de esta. Conocer, entender y confrontar las perspectivas de los actores que participan de esta escena práctica, facilitará la comprensión de la estructura y el funcionamiento real de esta práctica y, en consecuencia, la comprensión de las dinámicas educativas actuales. Lo que permitiría adoptar una posición crítica bien fundamentada que busque la consolidación de prácticas pedagógicas significantes.

\section{REFERENCIAS BIBLIOGRÁFICAS}

ALBERTO UTP (2018). "Entretien $\mathrm{N}^{\circ} 1$. 'Entretiens personneladministratif', annexe 2.1”. En Approche sémiotique de l'intégration des TIC et de leurs usages dans l'enseignement universitaire colombien, L. Liñan Durán, 5-10. Thèse de doctorat inédite. Limoges: Université de Limoges.

ALEXANDER USTA (2018). "Entretien N5. 'Entretiens personneladministratif', Annexe 2.5". En Approche sémiotique de l'intégration des TIC et de leurs usages dans l'enseignement universitaire colombien, L. Liñan Durán, 59-72. Thèse de doctorat inédite. Limoges: Université de Limoges.

BEATRIZ UdeA (2018). "Entretien N³. 'Entretiens personneladministratif', annexe 2.3”. En Approche sémiotique de l'intégration des TIC et de leurs usages dans l'enseignement universitaire colombien, L. Liñan Durán, 30-49. Thèse de doctorat inédite. Limoges: Université de Limoges.

BERTRAND, D. (2006). Glossaire de semiotique. http://denisbertrand. unblog.fr/glossaire-de-semiotique/ [26/06/2019].

CECILIA, U. (2018). “Entretien N². 'Entretiens personnel-administratif', annexe 2.2". En Approche sémiotique de l'intégration des TIC et de leurs usages dans l'enseignement universitaire colombien, L. Liñan Durán, 16-29. Thèse de doctorat inédite. Limoges: Université de Limoges. 
FONTANILLE, J. (2003). Sémiotique $d u$ discours. Limoges: PULIM / Université de Limoges (col. Nouveaux Actes Sémiotiques). (2016). Prácticas semióticas. Lima: Fondo Editorial Universidad de Lima.

GREIMAS, A. J. et COURTES, J. (1993). Sémiotique: dictionnaire raisonné de la théorie du langage. Paris: Hachette Supérieur.

KAMBOUCHNER, D.; MEIRIEU, P.; STIEGLER, B. et alii (2012). L'école, le numérique et la société qui vient. Paris: Mille et une nuits.

LANDOWSKI, E. (1993). "Étapes en socio-sémiotique”. En L'esprit de société: vers une anthropologie sociale du sens, 105-128. Liège: Editions Mardaga.

(2005). Les interactions risquées. Limoges: PULIM, Université de Limoges.

(2012). “Habría que rehacer la semiótica?”. Contratexto 20, $127-$ 155.

(2015). "Régimes de sens et formes d'éducation". Colloque Sémiotique et sciences humaines et sociales: la sémiotique face aux défis sociétaux du XXIe siècle, Limoges, 25-27 novembre, http://www.unilim.fr/colloquesemiodefisshs/wp-content/uploads/ sites/16/2015/11/Educ.Landowski.28oct.pdf [26/06/2019].

PIGNIER, N. (2016a). "Approche sémiotique des interactions dans les dispositifs d'enseignement à distance. Le cas de la plateforme Moodle". Colloque Interactions Multimodales Par ÉCran. Lyon: Institut Français de l'Éducation (IFé) ENS de Lyon.

(2016b). "L'éducation critique aux médiations informationnelles et communicationnelles en milieu numérique". Colloque L'éducation critique aux médias en contexte numérique. Paris: CEMTI, Université Paris 8.

SEMPRINI, A. (1994). "Sujet, interaction, mondes. Le lieu commun comme déixis instituante". Protée 22, http://constellation.uqac. ca/2353/1/Vol_22_no_2.pdf[26/06/2019].

Recibido el 15 de mayo de 2019.

Aceptado el 27 de junio de 2019. 